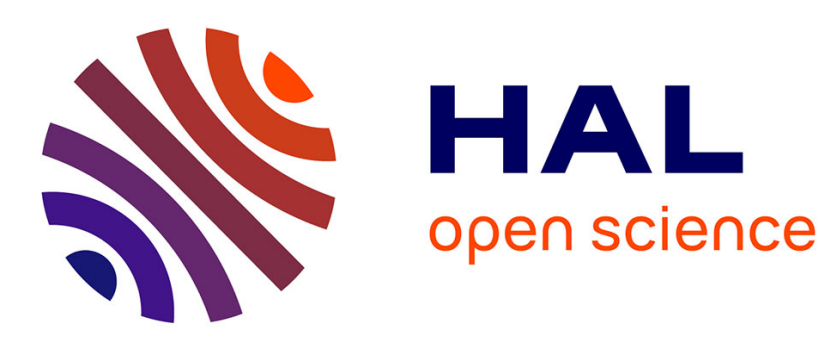

\title{
Recursive Bayesian estimation of the acoustic noise emitted by wind farms
}

Baldwin Dumortier, Emmanuel Vincent, Madalina Deaconu

\section{To cite this version:}

Baldwin Dumortier, Emmanuel Vincent, Madalina Deaconu. Recursive Bayesian estimation of the acoustic noise emitted by wind farms. 2017 IEEE International Conference on Acoustics, Speech, and Signal Processing (ICASSP)., Mar 2017, New Orleans, United States. hal-01428962

\section{HAL Id: hal-01428962 \\ https://hal.inria.fr/hal-01428962}

Submitted on 6 Jan 2017

HAL is a multi-disciplinary open access archive for the deposit and dissemination of scientific research documents, whether they are published or not. The documents may come from teaching and research institutions in France or abroad, or from public or private research centers.
L'archive ouverte pluridisciplinaire HAL, est destinée au dépôt et à la diffusion de documents scientifiques de niveau recherche, publiés ou non, émanant des établissements d'enseignement et de recherche français ou étrangers, des laboratoires publics ou privés. 


\title{
RECURSIVE BAYESIAN ESTIMATION OF THE ACOUSTIC NOISE EMITTED BY WIND FARMS
}

\author{
Baldwin Dumortier $^{1,2,3}$, Emmanuel Vincent ${ }^{1,2,3}$ and Madalina Deaconu ${ }^{1,3,4}$ \\ ${ }^{1}$ Inria, Villers-lès-Nancy, F-54600, France \\ ${ }^{2}$ CNRS, LORIA, UMR 7503, Villers-lès-Nancy, F-54600, France \\ ${ }^{3}$ Université de Lorraine, LORIA, UMR 7503, Villers-lès-Nancy, F-54600, France \\ ${ }^{4}$ Institut Elie Cartan de Lorraine - UMR 7502
}

\begin{abstract}
Wind turbine noise is often annoying for humans living in close proximity to a wind farm. Reliably estimating the intensity of wind turbine noise is a necessary step towards quantifying and reducing annoyance, but it is challenging because of the overlap with background noise sources. Current approaches involve measurements with on/off turbine cycles and acoustic simulations, which are expensive and unreliable. This raises the problem of separating the noise of wind turbines from that of background noise sources and coping with the uncertainties associated with the source separation output. In this paper we propose to assist a black-box source separation system with a model of wind turbine noise emission and propagation in a recursive Bayesian estimation framework. We validate our approach on real data with simulated uncertainties using different nonlinear Kalman filters.
\end{abstract}

Index Terms - Audio source separation, nonlinear Kalman filtering, uncertainties, wind turbine noise

\section{INTRODUCTION}

Wind energy is one of the most used sustainable energies in the world. The stochastic nature of wind raises numerous research problems including control [1], short-term wind prediction [2,3], resource assessment [4] or power curve estimation $[5,6]$. Among the limiting factors of the electric production, acoustic annoyance has been somewhat left behind in the prediction and control field. Yet, complaints from inhabitants can lead to severe penalties for the owners that must ensure the compliance with the applicable law of the country where the farm is set. In all countries acoustic annoyance is quantified by either the total noise level resulting from all acoustic sources which is called ambient noise, or by the acoustic emergence [7]. The acoustic emergence is the difference between the ambient noise level and the background noise level due to other noise sources besides the turbines. It can be seen as the acoustic gain brought by the use of wind turbines.

The acoustic emergence is difficult to estimate because both the propagation of the sound of the wind turbines [8] and the background noise rapidly fluctuate in an outdoor context. Nowadays, numerical simulation and measurement campaigns are used for that purpose but the calculated emergence is often inaccurate because of the sensitiveness of the acoustic variables to the fine topology and meteorological conditions $[9,10]$. To address this problem, the iEar project ${ }^{1}$ introduced an online control system [11] based on permanent sound level meters that estimate the intensity of wind turbine noise and background noise in real time by means of a proprietary audio source separation system using audio input only. The output of this source separation system is not perfect, though, since wind turbine noise and background noise often exhibit similar spectral and spatial characteristics that make them difficult to separate. Uncertainties associated with source separation have received some interest for speech and speaker recognition [12-16], but they have not been studied for environmental sounds yet, to the best of our knowledge.

In this paper, we propose a recursive Bayesian estimation framework for estimating the intensity of wind turbine noise and background noise that jointly takes into account the output of a source separation system (considered here as a black box) and a model of wind turbine noise emission and propagation. Our approach has the advantage of considering simultaneously the sound level meter measurements, the noise emission values provided by turbine manufacturers, and the results of audio source separation and acoustic simulation. We evaluate the suitability of different nonlinear Kalman filters to solve the estimation problem. Since the ground truth is unknown in real conditions, we validate our approach on real data obtained from an acoustic compliance study of a selected wind farm to which we added simulated uncertainties.

The rest of this paper is organised as follows. In Section 2 , we present the background and the notations. Then we present the proposed recursive Bayesian model in Section 3. In Section 4, we present the conducted experiments with nonlinear Kalman filters tested on semi-simulated data. Finally perspectives and discussions are given in Section 5.

\footnotetext{
${ }^{1}$ http://www.venathec.com/fr/iear
} 


\section{BACKGROUND AND NOTATIONS}

\subsection{Acoustic variables}

We assume that acoustical engineers have set sound level meters in different locations indexed by $j \in\{1, \ldots, J\}$. Each sound level meter measures the ambient noise $b_{t, j}$, i.e. the total noise level at location $j$ and time $t$ in A-weighted decibels (dBA). Each time $t$ corresponds to a period of $10 \mathrm{~min}$.

The ambient noise is the sum of the wind turbine noise $l_{t, j}$ and the background noise $r_{t, j}$ at location $j$ and time $t$. These two signals are assumed to be uncorrelated and to sum up on a linear scale $\left(\mathrm{W} / \mathrm{m}^{2}\right)$ [17] which gives in $\mathrm{dBA}$ :

$$
b_{t, j}=10 \log _{10}\left(10^{\frac{l_{t, j}}{10}}+10^{\frac{r_{t, j}}{10}}\right) .
$$

Furthermore, the acoustic emergence $e_{t, j}$ is defined as

$$
e_{t, j}=b_{t, j}-r_{t, j}
$$

This quantity represents the increase of the noise level due to the wind turbines and it has the meaning of an a posteriori signal-to-noise ratio.

\subsection{Acoustic model}

Besides acoustic measurements, acoustic simulations are conducted to predict the wind turbine noise $l_{t, j}$ with the help of the acoustic noise emission curves of the turbine manufacturer. These simulations are conducted by means of specific software such as Datakustik cadnaA ${ }^{2}$, that uses ray-tracing to simulate the propagation. To reduce the number of simulations, the variables $c_{t}$ and $o_{t}$ that respectively represent the turbine command variables (pitch angle, etc) and the meteorological variables (wind speed and direction), are reduced to a small number of number of discrete values denoted by $\mathcal{C}=\left\{c_{1}, \ldots, c_{N}\right\}$ and $\mathcal{O}=\left\{o_{1}, \ldots, o_{N}\right\}$ and the following acoustic formula is generally considered [18]:

$$
l_{t, j} \simeq 10 \log _{10}\left(\sum_{i}^{I} 10^{\frac{a_{o_{t}, i, j}}{10}} 10^{\frac{x_{c_{t}, o_{t}, i}}{10}}\right) .
$$

where $i \in\{1, \ldots, I\}$ indexes the wind turbines, $x_{c_{t}, o_{t}, i}$ is the noise emitted by turbine $i$ in dBA that both depends on the command $c_{t}$ in use and on the meteorological conditions $o_{t}$, and $a_{o_{t}, i, j}$ is an attenuation factor between wind turbine $i$ and sound level meter $j$ which depends on the meteorological conditions $o_{t}$ only.

\subsection{Measurements and uncertainties}

Finally, we assume that a source separation system separates the wind turbine noise and background noise signals and produces an estimate of the background noise level at each location and time. Neither the acoustic model nor the sound

\footnotetext{
${ }^{2}$ http://www.datakustik.com/en/products/cadnaa
}

level measurements and the source separation outputs are exact: they suffer from uncertainties. To distinguish them from the ground truth values, we adopt the following notations.

$b_{t, j}^{*}$ and $r_{t, j}^{\bullet *}$ respectively denote the sound level measurement of $b_{t, j}$ and the audio source separation estimate of $r_{t, j}$. They are stacked across locations into $J \times 1$ vectors $\mathbf{b}_{t}^{*}$ and $\mathbf{r}_{t}^{* \bullet} . x_{c_{t}, o_{t}, i}, a_{o_{t}, i, j}$, and $r_{t, j}$ respectively denote the real values of turbine noise emission, propagation, and background noise. They are stacked across turbines and/or locations into an $I \times 1$ vector $\mathbf{x}_{t}$, an $I J \times 1$ vector $\mathbf{a}_{t}$, and a $J \times 1$ vector $\mathbf{r}_{t}$. We denote by $\mathbf{z}_{t}=\left[\mathbf{b}_{t}^{* \mathrm{~T}} \mathbf{r}_{t}^{* \bullet \mathrm{T}}\right]^{\mathrm{T}}$ the overall $2 J \times 1$ observation vector and by $\mathbf{y}_{t}=\left[\mathbf{x}_{t}^{\mathrm{T}} \mathbf{a}_{t}^{\mathrm{T}} \mathbf{r}_{t}^{\mathrm{T}}\right]^{\mathrm{T}}$ the overall $(I J+I+J) \times 1$ state vector. Finally, $\mathbf{w}_{\mathbf{v}}$ denotes the uncertainty related to a given variable $\mathbf{v}$, that is the deviation from the ground truth.

\section{BAYESIAN ESTIMATION}

The aim of the proposed Bayesian approach is to refine the prior distribution resulting from the acoustic model thanks to the observations that comprise of the measured ambient noise and the outputs of the source separation system. The posterior distribution is then inferred thanks to the Bayes theorem [19]. In order to define the likelihood and the prior distribution, we model the uncertainties of the system as Gaussian random variables. This assumption is suitable since wind turbine manufacturers, sound level meter manufacturers, and simulation software systematically quantify uncertainties using standard deviations on the dBA scale.

\subsection{Prior}

For the prior, we use a first order auto-regressive moving average with external output vectors (ARMAX) model [20] for the emission, the propagation and the background noise:

$$
\mathbf{y}_{t}=\left[\begin{array}{c}
\mathbf{x}_{t} \\
\mathbf{a}_{t} \\
\mathbf{r}_{t}
\end{array}\right]=\left[\begin{array}{c}
\mathbf{F}_{\mathbf{x}} \mathbf{x}_{t-1}+\mathbf{G}_{\mathbf{x}} \mathbf{m}_{\mathbf{x}_{t}+\mathbf{w}_{\mathbf{x}_{t}}} \\
\mathbf{F}_{\mathbf{a}} \mathbf{a}_{t-1}+\mathbf{G}_{\mathbf{a}} \mathbf{m}_{\mathbf{a}_{t}}+\mathbf{w}_{\mathbf{a}_{t}} \\
\mathbf{F}_{\mathbf{r}} \mathbf{r}_{t-1}+\mathbf{G}_{\mathbf{r}} \mathbf{m}_{\mathbf{r}_{t}}+\mathbf{w}_{\mathbf{r}_{t}}
\end{array}\right] .
$$

This model takes into account both the temporal correlations (via the matrices $\mathbf{F}_{\mathbf{x}}, \mathbf{F}_{\mathbf{a}}$, and $\mathbf{F}_{\mathbf{r}}$ ) and the mean values $\mathbf{m}_{\mathbf{x}_{t}}$, $\mathbf{m}_{\mathbf{a}_{t}}$, and $\mathbf{m}_{\mathbf{r}_{t}}$ derived from the acoustic studies (via the matrices $\mathbf{G}_{\mathbf{x}}, \mathbf{G}_{\mathbf{a}}$, and $\mathbf{G}_{\mathbf{r}}$ ). The matrices $\mathbf{F}_{\mathbf{x}}, \mathbf{F}_{\mathbf{a}}, \mathbf{F}_{\mathbf{r}}, \mathbf{G}_{\mathbf{x}}, \mathbf{G}_{\mathbf{a}}$, and $\mathbf{G}_{\mathbf{r}}$ can be determined by learning in principle, even though we haven't tried this yet and we chose a simple random walk model for $\mathbf{r}_{t}\left(\mathbf{F}_{\mathbf{x}}=\mathbf{0}\right)$ as well as simple model for the propagation $\left(\mathbf{G}_{\mathbf{x}}=\mathbf{0}\right.$ and $\left.\mathbf{G}_{\mathbf{a}}=\mathbf{0}\right)$.

\subsection{Likelihood}

For the likelihood, we consider the following model:

$$
\begin{aligned}
& b_{t, j}^{*}=10 \log _{10}\left(\sum_{i}^{I} 10^{\frac{a_{t, i, j}}{10}} 10^{\frac{x_{t, i}}{10}}+10^{\frac{r_{t, j}}{10}}\right)+w_{b_{t, j}^{*}} \\
& r_{t, j}^{* \bullet}=r_{t, j}+w_{r_{t, j}^{\bullet}}+w_{b_{t, j}^{*}} .
\end{aligned}
$$


We model the ambient noise $b_{t, j}^{*}$ measured by the sound level meter at location $j$ as a Gaussian deviation from the real ambient noise $b_{t, j}$ expressed as a nonlinear function of the state variables with (1)-(3). Similarly, we model the background noise $r_{t, j}^{* \bullet}$ estimated by the source separation algorithm as a Gaussian deviation from the true $r_{t, j}$. The source separation uncertainty $w_{r_{t, j}^{*},}$, and the measurement uncertainty $w_{b_{t, j}^{*}}$ are strongly correlated with each other since source separation is performed from the same sound level meter input. Therefore the overall observation covariance is given by:

$$
\boldsymbol{\Sigma}_{\mathbf{z}}=\left[\begin{array}{cc}
\sigma_{b}^{* 2} \boldsymbol{I}_{J} & \sigma_{b}^{* 2} \boldsymbol{I}_{J} \\
\sigma_{b}^{* 2} \boldsymbol{I}_{J} & \left(\sigma_{b}^{* 2}+\sigma_{r}^{\bullet 2}\right) \boldsymbol{I}_{J}
\end{array}\right]
$$

with $\boldsymbol{I}_{J}$ the identity matrix of size $J$.

\subsection{Recursive Bayesian model}

The proposed formulation now fits with the recursive Bayesian filtering framework. We can summarize (4)-(6) with the general recursive Bayesian estimation model

$$
\begin{aligned}
\text { (prior) } & \mathbf{y}_{t}=\mathbf{F y}_{t-1}+\mathbf{G m}_{\mathbf{y}_{t}}+\mathbf{w}_{\mathbf{y}_{t}} \\
\text { (likelihood) } & \mathbf{z}_{t}=h\left(\mathbf{y}_{t}\right)+\mathbf{w}_{\mathbf{z}_{t}}
\end{aligned}
$$

with $h$ the nonlinear function in (5)-(6), and recursively infer the posterior distribution of the state variables by the general filtering equations

$$
\begin{aligned}
p\left(\mathbf{y}_{t} \mid \mathbf{z}_{1: t-1}\right) & =\int p\left(\mathbf{y}_{t} \mid \mathbf{y}_{t-1}\right) p\left(\mathbf{y}_{t-1} \mid \mathbf{z}_{1: t-1}\right) d \mathbf{y}_{t-1} \\
p\left(\mathbf{y}_{t} \mid \mathbf{z}_{1: t}\right) & \propto p\left(\mathbf{z}_{t} \mid \mathbf{y}_{t}\right) p\left(\mathbf{y}_{t} \mid \mathbf{z}_{1: t-1}\right)
\end{aligned}
$$

with $\mathbf{z}_{1: t}=\left\{\mathbf{z}_{1}, \ldots, \mathbf{z}_{t}\right\}$ the set of observations up to time $t$.

\section{EXPERIMENTS AND RESULTS}

\subsection{Data}

We validated our approach on data collected by VENATHEC SAS on a wind farm located in the north-east of France. For privacy reasons, the name and exact location of the wind farm are not mentioned. The wind farm is composed of 6 wind turbines (Vestas V112 - 3.3 MW) and 5 measurement locations were set up. In this paper, we consider $19 \mathrm{~h}$ of measurements that correspond to 115 time frames of $10 \mathrm{~min}$.

Since the ground truth is unknown in real conditions, we generated the test data as follows. For background noise, we considered the real series $\mathbf{r}_{t}$ measured when the turbines are stopped. For noise emission and propagation, we considered the mean values $\mathbf{m}_{\mathbf{x}_{t}}$ and $\mathbf{m}_{\mathbf{a}_{t}}$ provided by the turbine manufacturer and cadnaA, respectively, and generated $\mathbf{x}_{t}$ and $\mathbf{a}_{t}$ by adding random Gaussian uncertainties $\mathbf{w}_{\mathbf{x}_{t}}$ and $\mathbf{w}_{\mathbf{a}_{t}}$. We then generated observations $\mathbf{z}_{t}$ using (9) with random Gaussian measurement and separation uncertainties $\mathbf{w}_{\mathbf{b}_{t}^{*}}$ and $\mathbf{w}_{\mathbf{r}_{t}^{\bullet}}$. Such a simulation procedure is common in the wind turbine acoustics community. The uncertainties were assumed to be temporally and spatially uncorrelated and independent of $i$ and $j$. Hence, we omit indices $i$ and $j$ in the following.

We generated two datasets. The first dataset contains 125 test cases with all 6 turbines and 5 measurement locations. The standard deviations $\sigma_{x}, \sigma_{a}$, and $\sigma_{r} \bullet$ of the uncertainties $\mathbf{w}_{\mathbf{x}_{t}}, \mathbf{w}_{\mathbf{a}_{t}}$, and $\mathbf{w}_{\mathbf{r}_{t}^{\bullet}}$ are chosen in $\{0.5,1.5,2.5,3.5,4.5\}$ and all combinations of values are considered. These are plausible values encountered in practice. In the second dataset, $\sigma_{x}$, $\sigma_{a}$, and $\sigma_{r}$ are fixed to 3.5 , but subsets of 1 to 6 turbines and 1 to 5 measurement locations are selected, resulting in 30 test cases. For both datasets, the standard deviation $\sigma_{b}^{*}$ of the measurement uncertainty $\mathbf{w}_{\mathbf{b}^{*}}$ is fixed to $1.5 \mathrm{~dB}$, that is the standardized value for Type 1 sound level meters [21].

\subsection{Tested nonlinear Kalman filters}

We processed these data using various nonlinear Kalman filters with the following values for the model parameters. We set $\mathbf{F}_{\mathbf{x}}=\boldsymbol{I}, \mathbf{F}_{\mathbf{a}}=\boldsymbol{I}, \mathbf{F}_{\mathbf{r}}=\mathbf{0}, \mathbf{G}_{\mathbf{x}}=\mathbf{0}, \mathbf{G}_{\mathbf{a}}=\mathbf{0}$, and $\mathbf{G}_{\mathbf{r}}=\boldsymbol{I}$. The covariance of $\mathbf{w}_{\mathbf{y}_{t}}$ was defined as

$$
\boldsymbol{\Sigma}_{\mathbf{y}}=\left[\begin{array}{ccc}
\sigma_{x}^{2} \boldsymbol{I}_{J} & \mathbf{0} & \mathbf{0} \\
\mathbf{0} & \sigma_{a}^{2} \boldsymbol{I}_{I J} & \mathbf{0} \\
\mathbf{0} & \mathbf{0} & \boldsymbol{\Sigma}_{\mathbf{r}}
\end{array}\right]
$$

with $\boldsymbol{\Sigma}_{\mathbf{r}}$ the covariance matrix of $\mathbf{r}_{t}-\mathbf{r}_{t-1}$ measured when the turbines are stopped. The values of $\mathbf{m}_{\mathbf{x}_{t}}, \mathbf{m}_{\mathbf{a}_{t}}, \sigma_{x}, \sigma_{a}$, and $\sigma_{r} \bullet$ were set as above. The assumptions behind these settings are also common in the wind turbine acoustics community.

The Kalman filter [22] implements the general recursive Bayesian filter in (10)-(11). It provides update rules for the posterior mean $\hat{\mathbf{y}}_{t}$ and covariance $\hat{\boldsymbol{\Sigma}}_{\mathbf{y}_{t}}$ of the state vector $\mathbf{y}_{t}$ given the sequence of observations $\mathbf{z}_{1: t}$ up to time $t$. In the case of a linear function $h$, these update rules are exact.

In our specific problem, the function $h$ is nonlinear, hence we evaluated three different approximations : the extended Kalman filter [23], the unscented Kalman filter [24] and the central difference Kalman filter [25]. The first one is the most common approximation: it relies on first-order Taylor development of $h$ around a single point, namely the estimated state mean. When the covariance is large or $h$ is strongly nonlinear it poorly approximates the posterior distribution and the estimation can be weak. The two other filters are ingenious improvements of the extended Kalman filter based on statistical linearisation. They approximate the prior distribution with a number of well-chosen points that are propagated through the nonlinearity to capture the mean and covariance. The two filters slightly differ in the choice of the points and the way they reconstruct the mean and covariance.

\subsection{Evaluation metrics}

We evaluated the results by means of the root mean square error (RMSE) between the estimated background noise value 
$\hat{\mathbf{r}}_{t}$ and the ground truth $\mathbf{r}_{t}$ and the log-likelihood of the ground truth $\mathbf{r}_{t}$ given the posterior distribution of the background noise, assumed to be Gaussian with mean $\hat{\mathbf{r}}_{t}$ (subvector of $\hat{\mathbf{y}}_{t}$ ) and covariance $\hat{\boldsymbol{\Sigma}}_{\mathbf{r}_{t}}$ (submatrix of $\hat{\boldsymbol{\Sigma}}_{\mathbf{y}_{t}}$ ). We also computed the RMSE and the log-likelihood of the emergence, where the ground truth $\mathbf{e}_{t}$ was obtained from $\mathbf{y}_{t}$ and the posterior mean $\hat{\mathbf{e}}_{t}$ and covariance $\hat{\boldsymbol{\Sigma}}_{\mathbf{e}_{t}}$ were obtained by propagating $\hat{\mathbf{y}}_{t}$ and $\hat{\boldsymbol{\Sigma}}_{\mathbf{y}_{t}}$ through the nonlinearity via the nonlinear Kalman filter under test.

\subsection{Results}

Tables 1 and 2 report the average RMSE on the background noise estimate and the emergence estimate. The former is similar to the intrinsic standard deviation of the sound level meter $\left(\sigma_{b}^{*}=1.5 \mathrm{~dB}\right)$, while the latter is much smaller due to the fact that the measurement uncertainty $\mathbf{w}_{\mathbf{b}_{t}^{*}}$ on $\mathbf{b}_{t}$ and $\mathbf{r}_{t}$ cancels when subtracting those two quantities from each other. It is also much smaller than the average uncertainty of the source separation system $(2.5 \mathrm{~dB})$. The central difference Kalman filter appears to perform best in terms of RMSE, however the posterior covariance is underestimated so that the unscented Kalman filter yields a better log-likelihood.

\begin{tabular}{|c|c|c|}
\hline Filter & RMSE & log-likelihood \\
\hline $\begin{array}{c}\text { source separation estimate } \\
\text { (without Kalman filter) }\end{array}$ & 3.24 & $2.50 \times 10^{5}$ \\
\hline extended filter & 1.63 & $2.42 \times 10^{5}$ \\
\hline unscented filter & 1.63 & $2.41 \times 10^{5}$ \\
\hline central difference filter & $\mathbf{1 . 6 1}$ & $2.40 \times 10^{5}$ \\
\hline
\end{tabular}

Table 1. Average RMSE and log-likelihood of the background noise estimate on the first dataset.

\begin{tabular}{|c|c|c|}
\hline Filter & RMSE & log-likelihood \\
\hline $\begin{array}{c}\text { source separation estimate } \\
\text { (without Kalman filter) }\end{array}$ & 2.87 & $4.2675 \times 10^{4}$ \\
\hline extended filter & 1.01 & $-0.69 \times 10^{5}$ \\
\hline unscented filter & 0.79 & $1.54 \times 10^{5}$ \\
\hline central difference filter & $\mathbf{0 . 7 6}$ & $-0.1 \times 10^{5}$ \\
\hline
\end{tabular}

Table 2. Average RMSE and log-likelihood of the emergence estimate on the first dataset.

Figure 1 shows the dependency of the RMSE on the uncertainties in the data and on the number of wind turbines and measurement locations. As expected, thanks to the fusion of the model uncertainties and the separation uncertainties operated by the nonlinear Kalman filter, the RMSE on the emergence estimate decreases with the uncertainties in the data and it is always smaller than the standard deviation of the separation system, except for $\left(\sigma_{x}, \sigma_{a}, \sigma_{r} \bullet\right)=(3.5,3.5,0.5)$ and $\left(\sigma_{x}, \sigma_{a}, \sigma_{r} \bullet\right)=(4.5,4.5,0.5)$ where the central difference approximation is poor. Also, the RMSE increases with the number of wind turbines and decreases with the number of measurement locations.

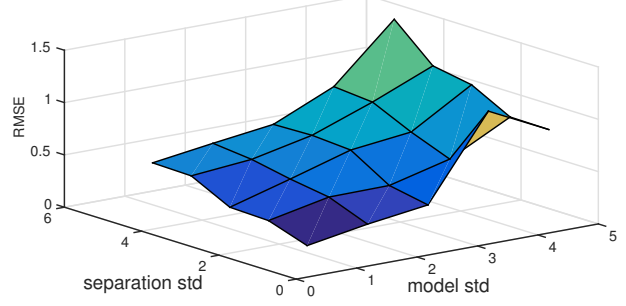

(a)

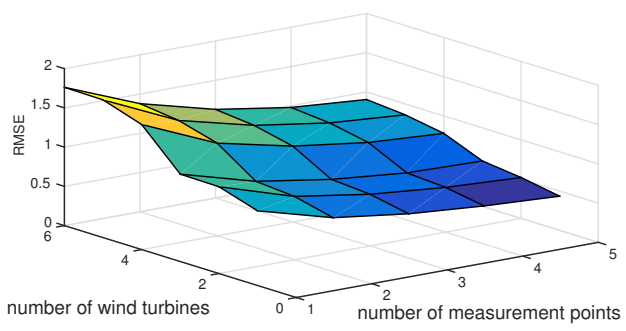

(b)

Fig. 1. RMSE of the emergence estimate as a function of (a) the standard deviations $\sigma_{x}$ and $\sigma_{a}$ of the model uncertainties and the standard deviation $\sigma_{r} \bullet$ of the source separation system on the first dataset and (b) the number of measurement locations and wind turbines on the second dataset. The central difference Kalman filter is used.

\section{CONCLUSION}

In this paper, we proposed a recursive Bayesian estimation framework adapted to the context of wind farms in order to improve a black-box source separation system. The proposed framework leverages the noise emission values provided by turbine manufacturers and the results of acoustic simulation. Our experiments showed that the central difference Kalman filter provides the lowest average RMSE on the emergence estimate and that this average RMSE is almost always smaller than the uncertainty of the source separation system alone. Future work includes addressing the poor performance of the central difference Kalman filter when the separation uncertainties are small and the model uncertainties are large, for instance by using iterated Kalman [26] or particle filters [27].

\section{ACKNOWLEDGEMENT}

This work was done with the collaboration and the support of VENATHEC SAS. In particular, the authors thank Jeremy Schild and Patrice Cornu from Venathec SAS for their implication. Experiments presented in this paper were carried out using the Grid'5000 testbed, supported by a scientific interest group hosted by Inria and including CNRS, RENATER and several Universities as well as other organizations (see https://www.grid5000.fr). 


\section{REFERENCES}

[1] Y. Guo, S. H. Hosseini, C. Y. Tang, J. N. Jiang, and R. G. Ramakumar, "An approximate wind turbine control system model for wind farm power control," Sustainable Energy, IEEE Transactions on, vol. 4, no. 1, pp. 262274, 2013.

[2] M. J. Holland and K. Ikeda, "Location robust estimation of predictive Weibull parameters in short-term wind speed forecasting," in Proc. ICASSP, 2015, pp. 39964000.

[3] A. Kuh and D. Mandic, "Applications of complex augmented kernels to wind profile prediction," in Proc. ICASSP, 2009, pp. 3581-3584.

[4] F. Bahlke, Y. Liu, and M. Pesamento, "Stochastic load scheduling for risk-limiting economic dispatch in smart microgrids," in Proc. ICASSP, 2016, pp. 2479-2483.

[5] M. Lydia, S. S. Kumar, G. E. P. Kumar, and A. I. Selvakumar, "Advanced algorithms for wind turbine power curve modeling," Sustainable Energy, IEEE Transactions on, vol. 4, no. 3, pp. 827-835, 2013.

[6] X. Liu, "An improved interpolation method for wind power curves," Sustainable Energy, IEEE Transactions on, vol. 3, no. 3, pp. 528-534, 2012.

[7] J. Schild and V. Chavand, "State of the art and new perspectives for the development of noise regulation of wind farms," Proc. 6th International Meeting on Wind Turbine Noise, 2015.

[8] C. Guarnaccia, N. E. Mastorakis, and J. Quartieri, "Wind turbine noise: theoretical and experimental study," International Journal of Mechanics, vol. 5, no. 3, pp. 129-137, 2011.

[9] C. Tickell, J. Ellis, and M. Bastasch, "Wind turbine generator noise prediction-comparison of computer models," in Proceedings of ACOUSTICS, 2004.

[10] G. Van den Berg, "Effects of the wind profile at night on wind turbine sound," Journal of Sound and Vibration, vol. 277, no. 4, pp. 955-970, 2004.

[11] B. Dumortier, E. Vincent, and M. Deaconu, "Acoustic control of wind farms," in Proc. Annual Conference of European Wind Energy Association (EWEA), 2015.

[12] D. Kolossa and R. Haeb-Umbach, Robust Speech Recognition of Uncertain or Missing Data, Springer, 2011.

[13] M. Delcroix, S. Watanabe, T. Nakatani, and A. Nakamura, "Cluster-based dynamic variance adaptation for interconnecting speech enhancement pre-processor and speech recognizer," Computer Speech and Language, vol. 27, no. 1, pp. 350-368, 2013.

[14] H. Kallasjoki, J. F. Gemmeke, and K. J. Palomki, "Estimating uncertainty to improve exemplar-based feature enhancement for noise robust speech recognition," IEEE Transactions on Audio, Speech, and Language Processing, vol. 22, no. 2, pp. 368-380, Feb 2014.

[15] D. T. Tran, E. Vincent, and D. Jouvet, "Nonparametric uncertainty estimation and propagation for noise robust ASR," IEEE/ACM Transactions on Audio, Speech, and Language Processing, vol. 23, no. 11, pp. 1835-1846, 2015.

[16] A. H. Abdelaziz, S. Watanabe, J. R. Hershey, E. Vincent, and D. Kolossa, "Uncertainty propagation through deep neural networks," in Proc. Interspeech, 2015.

[17] C. H. Hansen and C. Sehrndt, "Fundamentals of acoustics," Occupational Exposure to Noise: Evaluation, Prevention and Control. World Health Organization, 2001.

[18] A. L. Rogers, J. F. Manwell, and S. Wright, "Wind turbine acoustic noise," Renewable Energy Research Laboratory, Amherst: University of Massachusetts, 2006.

[19] C. Bishop, Pattern Recognition and Machine Learning, Springer, New York, 2nd edition, 2007.

[20] D. P. Bertsekas, Dynamic Programming: Deterministic and Stochastic Models, Academic Press, 1976.

[21] International Electrotechnical Commission, "IEC 61672-1: 2002 Electroacoustics-Sound level metersPart 1," 2002.

[22] T. Kailath, A. H. Sayed, and B. Hassibi, Linear estimation, vol. 1, Prentice Hall, 2000.

[23] S. S. Haykin et al., Kalman filtering and neural networks, Wiley Online Library, 2001.

[24] S. J. Julier and J. K. Uhlmann, "New extension of the Kalman filter to nonlinear systems," in Proc. AeroSense, 1997, pp. 182-193.

[25] R. Van Der Merwe, Sigma-point Kalman filters for probabilistic inference in dynamic state-space models, Ph.D. thesis, Oregon Health \& Science University, 2004.

[26] G. Sibley, G. S. Sukhatme, and L. Matthies, "The iterated sigma point Kalman filter with applications to long range stereo.," in Robotics: Science and Systems, 2006, vol. 8, pp. 235-244.

[27] B. Ristic, S. Arulampalam, and N. Gordon, Beyond the Kalman filter: Particle filters for tracking applications, vol. 685, Artech house Boston, 2004. 